\title{
Molecular detection of rabies encephalitis and correlation with cytokine expression
}

\author{
Gerard J Nuovo ${ }^{1}$, Dulcelena L DeFaria ${ }^{2}$, Juan G Chanona-Vilchi ${ }^{3}$ and Yilan Zhang ${ }^{1}$ \\ ${ }^{1}$ Department of Pathology, The Ohio State University, Columbus, OH, USA; ${ }^{2}$ Department of Pathology, Santa \\ Casa de Sáo Paulo Hospital, Sáo Paulo, Brazil and ${ }^{3}$ Department of Pathology, Instituto Nacional de \\ Concerologia, Mexico City, Mexico
}

\begin{abstract}
The purposes of this study were to elucidate the role of cytokine upregulation in the pathogenesis of rabies encephalitis and to compare the detection of Negri bodies with that of rabies protein by immunohistochemistry and rabies RNA by reverse transcriptase (RT) in situ PCR for its diagnosis. Negri bodies were evident in 4/7 of the documented rabies cases; viral protein and viral RNA were detected in each case. The average number of rabies-infected cells, determined by counting $\mathbf{1 5 0}$ neurons in serial sections in areas where viral protein was evident, with the three different detection methods was: Negri bodies $(<1 / 150)$, immunohistochemistry $(4 / 150)$, and RT in situ PCR (49/150). No rabies protein or RNA was detected in four control brain tissues that were read with the rabies cases in a blinded fashion. The ratio of cells expressing tumor necrosis factor $\alpha$ (TNF $\alpha$ ) or inducible nitric oxide synthetase (iNOS) to $1 \mathrm{SSI}-1 / \mathrm{SOCS}-1$ (suppressors of cytokine signaling) expression, which is a novel class of negative feedback regulators of cytokine receptor signaling, was markedly increased only in the areas where many viral infected cells were present. Colabeling experiments showed that most of the cells expressing iNOS or TNF $\alpha$ were not virally infected, but rather adjacent to rabies-infected neurons. We conclude that RT in situ PCR for rabies virus is the most accurate test for the determination of viral load in rabies encephalitis. Further, the disease is characterized by massive viral infection of neurons in a markedly focal distribution in conjunction with a concomitant upregulation of cytokine expression in adjacent, noninfected cells that may be due, in part, to SOCS downregulation.
\end{abstract}

Modern Pathology (2005) 18, 62-67, advance online publication, 24 September 2004; doi:10.1038/modpathol.3800274

Keywords: colabeling; cytokine; immunohistochemistry; in situ PCR; rabies

Rabies encephalitis is an acute infectious disease of the central nervous system caused by a RNA virus of the Lyssa group. ${ }^{1}$ The major reservoirs of the disease in the United States are raccoons, skunks, and bats; in the latter case, the animal may be infectious for long periods of time and not appear ill. ${ }^{1-5}$ Although the virus is most commonly transmitted by bites, it has been shown that rabies may be acquired by inhaling infected excreta of bats or, rarely, from transmission in the laboratory setting. ${ }^{1-5}$

The incubation period for rabies encephalitis is broad, ranging from a few weeks to 1 year that relates to several variables including the site of the inoculum and the viral load associated with the trauma. $^{1,3}$ The replication of viruses occurs in the cytoplasm of infected cells at the site of inoculation

Correspondence: Dr G Nuovo, MD, Ohio State Medical Center, Dept Pathology -Div Anatomic Pathology, S305 Rhodes Hall; 450 W 10th Ave, Columbus, OH 43210-1228, USA.

E-mail: nuovo-1@medctr.osu.edu

Received 24 May 2004; revised and accepted 3 August 2004; published online 24 September 2004
1-3 days after the trauma and is then transported by axons of peripheral nerves to the CNS where it replicates within the cytoplasm of neurons. ${ }^{1,3,5}$ This suggests that viral spread may show a fascicle-type pattern in the central nervous system, reflecting the histologic distribution of nerve bundles that correspond to the proximal transmission from the site of inoculum. Rapid diagnosis of the infection in the donor animal is critical in order to allow sufficient time for the vaccine to become effective in the recipient.

The histologic correlates in the central nervous system of rabies encephalitis are relatively mild, considering the marked clinical symptomatology. The pathognomonic finding is the Negri body which are round to oval acidophilic intracytoplasmic inclusions that tend to be more numerous in pyramidal cells of hippocampus and Purkinje cells of the cerebellum. ${ }^{1-5}$ However, Negri bodies are inconstantly present and may not be detectable in $20-50 \%$ of cases of rabies encephalitis. ${ }^{1,5}$ Corroboration of rabies encephalitis may be done by immunohistochemistry, electron microscopy, mice 
inoculation, and reverse-transcriptase polymerase chain reaction (RT-PCR); ${ }^{1-5}$ the latter method, although sensitive, is best done with unfixed, infectious material and does not allow direct correlation with the histologic findings.

The exact mechanism whereby rabies causes fatal encephalitis is not clear; viral-induced neuronal cell lysis appears to be a rare event. Rabies-induced synthesis of inducible nitric oxide (iNOS) in the central nervous system has been suggested as a pathogenetic step in neuronal cell damage in rabies infections. ${ }^{6,7}$ Tumor necrosis factor $\alpha$ (TNF $\alpha$ ) expression has also been suggested as an important factor in rabies encephalitis, both in terms of direct toxicity and as modulating viral production..$^{8-10}$ However, the studies on TNF $\alpha$ and iNOS expression have been done on animal models, and it is not clear if these data can be extrapolated to human rabies. Other viral encephalitis in humans, such as from HIV-1 infection, are marked by massive viral infection with concomitant expression of a variety of chemicals, including iNOS, TNF $\alpha$, and a variety of interleukins. ${ }^{11,12}$ Viral production appears to be synergistic with the production of cytokines such as $\mathrm{TNF} \alpha$ that can cause neuronal damage, in that cytokine expression is mostly evident in noninfected cells present adjacent to cells which contain the virus. ${ }^{11,12}$ The purpose of this study is to examine different diagnostic methodologies (histology, immunohistochemistry, and RT in situ PCR) for the detection of rabies encephalitis, and to elucidate the pathogenesis of this fatal disease by correlating viral production with cytokine expression.

\section{Materials and methods}

\section{Case Selection}

Seven cases of rabies encephalitis in humans were studied; five of these cases were from Brazil and the other two from the United States. We also studied three cases of fatal rabies encephalitis in $\operatorname{dogs}^{2}$ or raccoons. ${ }^{1}$ Diagnosis of these rabies cases was done by clinical history with corroboration by immunohistochemistry. Two other cases of fatal encephalitis in humans were studied where rabies infection was in the differential diagnosis; both occurred in the United States and were marked by rapidly progressive encephalitis in a 4-year-old boy and 22-year-old man, respectively, with possible exposure to bites by bats. In all cases, two to four formalin-fixed paraffinembedded tissues from either the midbrain, cerebellum, hippocampus, or cerebrum were studied.

\section{Viral Analysis}

Histologic sections that were stained with hematoxylin and eosin were examined in each case for Negri bodies. The average number of cells with Negri bodies was determined by counting 150 neurons in sections of Ammon's horn of the hippocampus or cerebellum. Two cases of histologically normal hippocampal tissue from two people who died suddenly of acute myocardial infarctions and two tissue sections of the hippocampus from people who died of AIDS-related dementia were included; the slides were read blinded to the clinical information.

Immunohistochemistry was done using a previously published protocol. ${ }^{11-14}$ The rabies antibody (DF A II, Light Diagnostics, Temecula, CA, USA), is fluoroscein-tagged and was detected using an antifluoroscein-peroxidase conjugate. The antibody against TNF $\alpha$ (Chemicon Corporation, CA, USA) was unlabeled and detected using the BioGenex (San Ramon, CA, USA) Supersensitive kit. Optimal conditions for each antibody were determined to be a dilution of 1:10 (rabies) and 1:50 (TNF $\alpha$ ) with predigestion in a protease solution (pepsin $2 \mathrm{mg} / \mathrm{ml}$ ) for 15-30 min.

Our RT in situ PCR protocol has been previously described. ${ }^{11-14}$ Briefly, optimal protease digestion time was determined using nonspecific incorporation of the reporter nucleotide digoxigenin dUTP. Optimal protease digestion is followed by overnight incubation in Rnase-free DNase (10 U per sample, Boehringer Mannheim, Indianopolis, IN, USA) and one-step RT/PCR using the rTth system and digoxigenin dUTP. The chromogen is nitroblue tetrazolium and bromochloroindolyl phosphate (NBT/ BCIP) with nuclear fast red as the counterstain. The primer sequences for TNF $\alpha$ and iNOS were previously published ${ }^{11,12}$ as were the primer sequences for suppressors of cytokine signaling (SOCS) expression. ${ }^{14}$ The primer sequences for the rabies virus were: GAGTCACTCGAATATGTC, GT ATTGCCTCTCTAGCGG, CTACAATGGATGCCGAC, GACATGTCCGGAAGACTG. These nested primers are specific for the nucleoprotein-related transcript and leader RNA region of the rabies virus as per the BLAST sequence analysis of the NCBI database. The immunohistochemical and RT in situ PCR studies were done on serial sections ( $4 \mu \mathrm{m}$ apart) from the sections studied by $\mathrm{H} \& \mathrm{E}$ to assure that the same groups of cells were examined. Controls for the RNA-based rabies signal included pretreatment of the tissue with RNase digestion and use of irrelevant primers (human papillomavirus related) as this virus cannot infect the brain. ${ }^{11,12}$ Additional controls for rabies, $\mathrm{TNF} \alpha$ and iNOS were sections of brain tissue from two people who died of acute myocardial infarctions, and had no clinical or histologic evidence of central nervous system malfunction.

\section{Results}

Seven cases of fatal rabies encephalitis in humans documented by clinical symptomatology and immunofluorescence were initially studied. From 1 to 4 formalin-fixed, paraffin-embedded sections 
were studied from each case for a total of 16 brain sections that included in each case Ammon's horn of the hippocampus and/or cerebellum as well as, in some cases, sections of the midbrain and cerebrum. The histologic changes in these 16 tissues ranged from no discernible pathologic changes to focal perivascular infiltrates of lymphocytes and monocytes as well as microglial nodules (Figure 1). A total of 20 tissues were analyzed for Negri bodies (the 16 tissues plus two sections of the hippocampus from two people who died of acute myocardial infarctions with no evidence of brain damage and two sections of hippocampus from two people who died of AIDS-dementia); the analysis was done blinded to the clinical information. Negri bodies were present in brain tissues from four of the seven cases (57\%) and in none of the four control central nervous system sections. Negri bodies were rare in the positive cases, with a range of $0-5$ such cytoplasmic inclusions per 150 large neurons in the hippocampus or cerebellum, with an average of less than 1/150 (data not shown).

Next, we compared detection of Negri bodies with the detection of rabies protein by immunohistochemistry in serial (subjacent) sections, to allow for direct comparison with the same groups of neurons. In each of the seven cases, rabies protein was detected in the sections of hippocampus and cerebellum. The range of positive large neurons per 150 cells was $1-15$ with a mean value of $4 / 150$ $(2 \%)$ with a standard deviation of 4 . Viral protein localized to the cytoplasm (Figure 1). The four control brain tissues were all negative for viral antigen.

In order to determine if the immunohistochemical data were an accurate representation of the viral load, defined by the number of infected neurons in a given field, RT in situ PCR analysis for viral RNA using nested primers was performed. This test was positive in each of the seven cases using serial section analysis and reading the slides blinded to the clinical information; the four control cases were all negative. The detection rate of rabies RNA in the hippocampus and cerebellum was much higher than for the viral protein with a range of 19-69 infected cells and a mean of $49 / 150$ large neurons (32\%) with a standard deviation of 13. The cytoplasmic-based signal was eliminated by pretreatment with RNase or substitution with human papillomavirus-specific primers, demonstrating that it was RNA-based (Figure 1).

It is important to stress that viral RNA distribution was very focal, with some areas in the hippocampus, cerebellum, midbrain, or cerebrum showing many infected cells and adjacent areas from 5 to $10 \mathrm{~mm}$ away in the same tissue block showing few to no infected cells. In each tissue positive for rabies viral RNA, the target localized to the cytoplasm or perinuclear area of small to large neurons and was not evident in either endothelial cells or the mononuclear/lymphocytic cells. Some infected cells had the cytologic appearance of astrocytes. Colabeling experiments with a phenotypic marker of astrocytes, GFAP, confirmed that rabies virus could infect such cells, but in far fewer numbers than infected neurons (Figure 2).

The three cases of rabies encephalitis in animals showed a similar poor sensitivity with Negri body detection and 10-fold increase in the number of positive cells in the large hippocampal neurons when comparing RT in situ PCR to immunohistochemistry (data not shown).

In the next part of the study, two cases of possible rabies encephalitis were analyzed. Each was obtained from the consult files of one of us (GJN) and, though Negri bodies were not evident on initial examination, was obtained from people with possible exposure to an animal (bat) bite. In neither case was viral protein detected by immunohistochemistry. However, many rabies-infected large neurons were noted in the area of the cerebellum in one of these cases. Additional tissue sections were obtained which did reveal rare Negri bodies. The other case was negative for rabies viral RNA; additional testing showed that there was widespread infection of the brain by rotavirus (localizing primarily to the endothelial cells) as was previously reported. ${ }^{13}$

In the final part of the study, the histologic distribution of rabies RNA was compared to that of iNOS, TNF $\alpha$ and SOCS-1 mRNA. There was a striking correlation between rabies RNA, iNOS and $\mathrm{TNF} \alpha$ expression; the latter two mRNAs were abundant in the exact same areas in the serial sections where rabies RNA was evident and were either rarely evident or not evident in the adjacent areas in the same tissue sections where viral RNA was not present (Figure 2). Cells expressing iNOS and $\mathrm{TNF} \alpha$ had the cytologic features of astrocytes and microglial cells and ranged in numbers from 10 to $33 / 150$ cells and from 5 to 24/150 cells (excluding neurons), respectively. In comparison, SOCS expression was rare in these areas of massive rabies virus infection with occasional cells (ranging from 3 to 15/150 cells (excluding neurons)) showing SOCS expression in the cytoplasm in areas where viralinfected cells were not seen (data not shown). Although, on cytologic examination, it appeared that the viral-infected cells were different from the cells expressing $\mathrm{TNF} \alpha$ or iNOS, colabeling experiments were done to more accurately address this issue. Colabeling for $\mathrm{TNF} \alpha \mathrm{RNA}$ and viral protein clearly demonstrated that most of the cells expressing $\mathrm{TNF} \alpha$ where viral-negative cells that were in close proximity to viral-infected neurons (Figure 2).

\section{Discussion}

The main findings of this study were twofold: the pathogenesis of rabies encephalitis includes a massive infection of neurons in a very focal distribution with concomitant overexpression of 

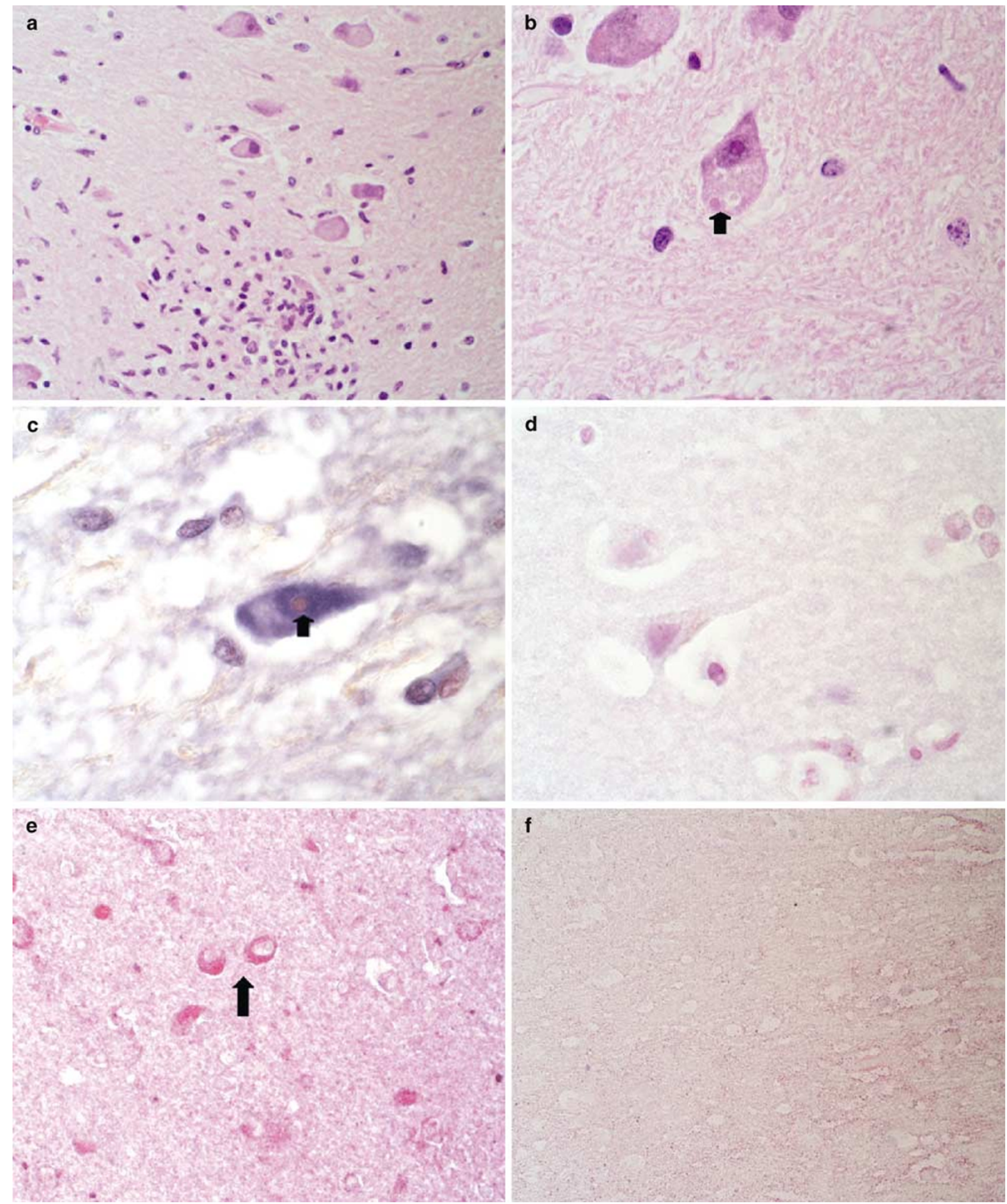

\section{f}

Figure 1 Viral and histologic correlates of rabies encephalitis. Panel (a) shows an area of Ammon's horn where a microglial nodule is evident, although such inflammatory-type changes are rare in rabies encephalitis. Panel (b) is a higher magnification of a large neuron in this area where a cytoplasmic inclusion (Negri body) is evident (arrow). PCR-amplified rabies cDNA was evident in the cytoplasm of many neurons in this area; panel (c) shows a high magnification so that the Negri body (arrow) is also visible. The negative controls each showed loss of signal and included pretreatment in RNase (panel d), omission of rabies-specific primers, substitution with irrelevant (HPV-specific) primers and testing of noninfected brain tissue (not shown). Panel (e) shows detection of rabies protein by immunohistochemistry; note the cytoplasmic-based red signal in the larger neurons. The negative controls each showed loss of signal and included omission of the primary antibody (panel f), and testing of noninfected brain tissue (not shown). 

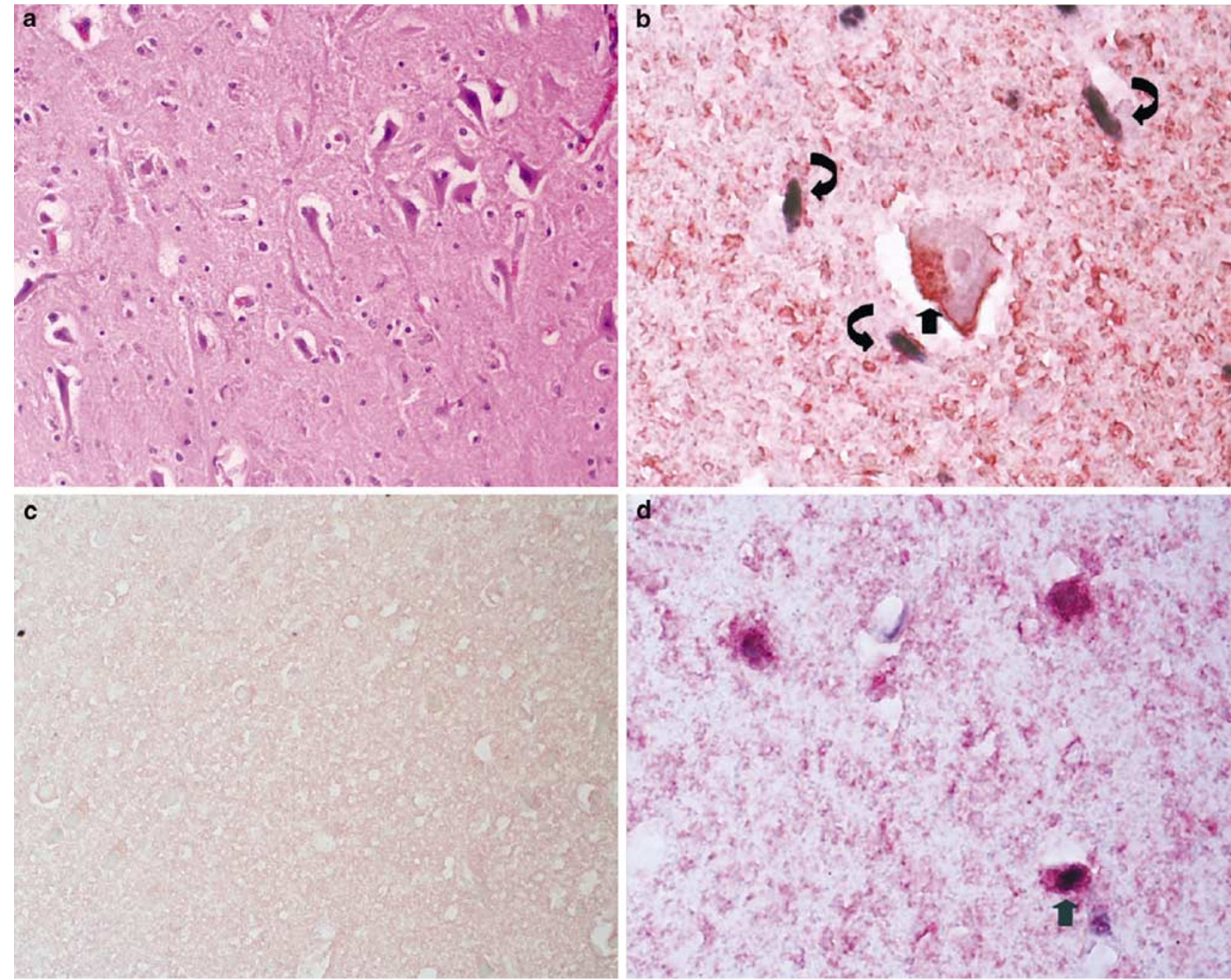

Figure 2 Viral and molecular correlates of rabies encephalitis. Panel (a) shows an area of Ammon's horn in which the histologic changes are minimal and nonspecific. Many rabies-infected cells were evident in this area with many cells expressing TNF $\alpha$ and iNOS. Colabeling experiments with rabies protein by immunohistochemistry (red signal, small arrow) and TNF $\alpha$ mRNA by RT in situ PCR (blue signal, large arrows) showed that the cells expressing TNF were usually not infected by the rabies virus but, rather, surrounded rabiesinfected neurons (panel (b)). Note that the cells expressing TNF $\alpha$ have the elongate phenotype characteristic of microglial cells. The signals were lost in the serial section if the tissue was pretreated with RNase, tested for TNF $\alpha$ mRNA expression, and then tested for rabies protein with omission of the primary antibody (panel (c)). Although most of the rabies-infected cells had the phenotype of neurons, occasional cells may have been astrocytes; colabeling experiments with GFAP (red signal) and rabies RNA (blue signal) documented rare infected astrocytes (panel (d), arrow).

TNF $\alpha$ and iNOS in neighboring noninfected cells and loss of expression of SOCS mRNA; detection of rabies cDNA after in situ amplification is much more accurate at determining the viral load (as defined by the number of infected neurons in a given area) than immunohistochemistry or histologic analysis for Negri bodies.

It is not surprising that intracytoplasmic inclusions (Negri bodies) are not a sensitive indicator of rabies virus infection, as noted by prior investigators. ${ }^{1-5}$ Most of the cases of viral infection associated with diagnostic inclusions/cytologic changes that are pathognomonic are associated with DNA viruses, such as cytomegalovirus, human papillomavirus, and herpes simplex virus, that cause, as expected, nuclear changes. Although infection with RNA viruses can produce inclusions characteristic of the disease, such as measles virus infection, in most cases, the inclusions are nonspecific and rarely seen. Indeed, based on the RT in situ PCR data, Negri bodies were found in less than $1 \%$ of infected neurons and, not surprisingly, were not detectable in several of our cases of both human and animal fatal rabies encephalitis. Immunohistochemistry was much more accurate in identifying rabiesinfected cells, but, nonetheless, detected only about $7 \%$ of the infected neurons based on the RT in situ PCR data done on serial ( $4 \mu \mathrm{m}$ distal) sections. There are several possible explanations for this observation. The rabies target protein may be produced in relatively small amounts or be, in part, masked by the formalin fixation protocol. Whether the detection rate with immunohistochemistry can be improved with analysis of different antigens, or by 
amplifying or unmasking procedures with the technique remains to be determined. Although immunohistochemistry did detect the rabies infection in each of the seven cases, it was negative in another case where rabies-infection was in the differential diagnosis and RT in situ PCR demonstrated many rabies-infected neurons. This suggests that additional testing for viral RNA with an in situ amplification process may be indicated in cases of suspected rabies infection where the immunohistochemical test is negative. Although solution phase RT-PCR would be an alternative assay, it is best done on unfixed, potentially infectious material and does not allow for correlation with the histologic findings. If rabies infection is a diagnostic possibility, we recommend fixing the tissue immediately in $10 \%$ buffered formalin or other related cross-linking fixative for $4-15 \mathrm{~h}$, as this is optimal for in situ detection. ${ }^{11,12}$

An interesting observation in this study was the striking focality of the rabies virus distribution, best appreciated with the RT in situ PCR method. This may reflect the pattern of spread from axons at the site of infection distally to series of neurons present in the downstream fascicles. As documented in other studies, the histologic inflammatory-cell correlates of this massive infection were relatively minimal, with rare and focal accumulations of lymphocytes and monocytes, typically around blood vessels. However, despite the relatively paucicellular response in the central nervous system to the infection, there was massive upregulation of both TNF $\alpha$ and iNOS, each of which can damage neurons and supporting tissue. ${ }^{7,10-12}$ This suggests a multifactorial pathogenic process for rabies encephalitis that includes widespread viral infection and concomitant increased expression of a variety of cellular factors as well as downregulation of SOCS expression. It is possible that cytokine expression may modulate rabies viral production in the central nervous system as suggested by others in animalbased studies, as well as contributing directly to the neuropathogenesis of the infectious process. ${ }^{7-10}$ It follows that inhibition of cytokine expression may have some therapeutic potential in this otherwise fatal disease, as has been demonstrated in a murine model $;^{9}$ this clearly awaits further study in humans. To our knowledge, this study is the first to document the histologic relationship of direct infection of rabies virus with expression of $\mathrm{TNF} \alpha$ and iNOS in people using an in situ-based assay.

It was interesting that cytokine expression in rabies encephalitis was found mostly in cells that were not virally infected, but rather adjacent to rabies-infected cells. This is similar to what has been described in AIDS-related dementia where a massive productive infection by HIV-1 is seen in concert with increased expression of iNOS, TNF $\alpha$, and other cytokines in the neighboring noninfected cells. ${ }^{11,12}$ Given that AIDS-related dementia is a slowly progressive disease and rabies encephalitis rapidly progressive, one may speculate that this process of viral infection upregulating cytokine expression in neighboring cells which, in turn, may modulate viral production may be an important process in viral encephalitis in general.

\section{Acknowledgements}

Supported by grants from the Lewis Foundation (GJN) and P30CA16058, National Cancer Institute, HL70574 (GJN).

\section{References}

1 Dupont JR, Earle KM. Human rabies encephalitis. A study of forty-nine fatal cases with a review of the literature. Neurology 1965;15:1023-1034.

2 Bahmanyar M, Fayaz A, Noorsalehi S, et al. Lightmicroscopical diagnosis of rabies. Lancet 1978;11: 302-303.

3 Depowski P, Trimarchi C, Qian, et al. A 54-year-old man presents with severe back pain. Arch Pathol Lab Med 2002;125:491-492.

4 Jackson AC, Ye H, Ridaura-San, et al. Quantitative study of the infection in brain neurons in human rabies. J Med Virol 2001;65:614-618.

5 Mrak RE, Young L. Rabies encephalitis in a patient with no history of exposure. Hum Pathol 1993;24: 109-110.

6 Ubol S, Sukwattanapan C, Maneerat Y. Inducible nitric oxide synthase inhibition delays death of rabies virusinfected mice. J Med Microbiol 2001;50:238-242.

7 Koprowski H, Zheng YM, Heber-Katz E, et al. In vivo expression of inducible nitric oxide synthase in experimentally induced neurologic diseases. Proc Natl Acad Sci USA 1993;90:3024-3027.

8 Camelo S, Lafage M, Lafon M. Absence of the p55 Kd TNF-alpha receptor promotes survival in rabies virus acute encephalitis. J Neurovirol 2000;6:507-518.

9 Camelo S, Castellanos J, Lafage $\mathrm{M}$, et al. Rabies virus ocular disease: T-cell-dependent protection is under the control of signaling by the p55 tumor necrosis factor alpha receptor, p55TNFR. J Virol 2001;75: 3427-3434.

10 Marquette C, Van Dam AM, Ceccaldi PE, et al. Induction of immunoreactive interleukin-1 beta and tumor necrosis factor-alpha in the brains of rabies virus infected rats. J Neuroimmunol 1996;68:45-51.

11 Nuovo GJ, Alfieri ML. AIDS dementia is associated with massive, activated HIV-1 infection and concomitant expression of several cytokines. Mol Med 1996;2:358-366.

12 Nuovo GJ, Gallery F, MacConnell P, et al. In situ detection of PCR-amplified HIV-1 nucleic acids and tumor necrosis factor RNA in the central nervous system. Am J Pathol 1994;144:659-666.

13 Morrison C, Gilson T, Nuovo GJ. Histologic correlates of fatal rotaviral infection. Hum Pathol 2001;32: 216-221.

14 Satosar A, Ramirez N, Davis J, et al. Histologic correlates of viral and bacterial infection of the placenta associated with severe morbidity and mortality in the newborn. Hum Pathol 2004;35:536-545. 